\section{ORIGINAL RESEARCH}

V.S. Oza

E. Wang

A. Berenstein

M. Waner

D. Lefton

J. Wells

F. Blei

\title{
PHACES Association: A Neuroradiologic Review of 17 Patients
}

BACKGROUND AND PURPOSE: We present neuroradiologic findings in 17 patients with posterior fossa malformations, hemangiomas, arterial anomalies, cardiac defects, eye abnormalities, and sternal or ventral defects (PHACES) association and identify those at highest risk of central nervous system (CNS) structural, cerebrovascular, and neurodevelopmental abnormalities.

MATERIALS AND METHODS: Patients with PHACES association were identified in the Vascular Anomalies Program at New York University Medical Center from 1998 to 2007. Many patients were followed in conjunction with other specialists at the Birthmark Institute at Roosevelt Hospital. Clinical records and imaging studies were reviewed retrospectively. Criteria for diagnosis of PHACES were based on previously published indicators. Imaging studies were independently re-reviewed by a neuroradiologist. Segmental mapping of cutaneous hemangioma distribution by photograph review and presence or absence of other PHACES-associated findings were correlated with radiologic findings.

RESULTS: Patients with large facial cutaneous (S1-S4) hemangiomas were especially at risk of CNS structural and cerebrovascular anomalies; S1 with ocular anomalies; and S3 with airway, ventral, and cardiac anomalies. All patients with CNS structural malformations had a cerebrovascular abnormality, and this cohort was at risk for developmental and/or other neurologic sequelae. Four patients had supratentorial CNS anomalies, including cortical dysgenesis and migration abnormalities. Some patients with CNS arteriopathy progressed to aneurysms.

CONCLUSION: Our data support and expand the work of others, identifying risk factors for segmental hemangiomas. In addition to posterior fossa CNS anomalies, supratentorial anomalies may be present in patients with PHACES, and this may correlate with significant clinical sequelae. The long-term prognosis of these patients remains unknown.

$\mathbf{P}$ osterior fossa malformations, segmental hemangiomas, arterial anomalies, cardiac defects, eye abnormalities, and sternal or ventral defects (PHACES) (Online Mendelian Inheritance in Man, 606519) are an association defined by segmental cutaneous hemangioma and malformations in the brain, neck, and/or thorax. The segmental pattern is a fairly new concept. ${ }^{1,2}$ Pascual-Castroviejo, in 1978, ${ }^{3}$ described 7 patients with facial hemangiomas and intracranial malformations. Other cases have since been reported-cutaneous hemangioma-vascular PHACES is the most widely accepted diagnostic label. ${ }^{4-7}$ Current literature suggests that PHACES may be present in up to $2 \%$ of children with facial hemangiomas and $20 \%$ of children with "segmental" facial hemangiomas. ${ }^{8}$ The prevalence may be even higher because many patients remain undiagnosed due to incomplete clinical and radiologic evaluation. Additionally, patients are often misdiagnosed as having other phakomatoses, such as Sturge-Weber syndrome. The etiology of PHACES remains ambiguous but may represent a field defect due to an insult in early embryonic development ${ }^{9}$

The hallmark of PHACES is a segmental hemangioma, usually facial. There is commonly incomplete phenotypic ex-

\section{Received July 14, 2007; accepted after revision October 29}

From the Departments of Pediatrics (V.S.O., F.B.), Radiology (E.W.), Surgery (Plastic) (F.B.), and Neurology (J.W.), New York University School of Medicine, New York, NY; Vascular and Birthmarks Institute of New York (A.B., M.W., D.L.), Roosevelt Hospital, New York, NY; and Albert Einstein School of Medicine (A.B., M.W., D.L.), Yeshiva University, New York, NY

Please address correspondence to Francine Blei, MD, Stephen D. Hassenfeld Center for Children with Cancer and Blood Disorders of NYU Medical Center, 160 East 32nd St, 2nd Floor, L3-Medical, New York, NY 10016; e-mail: francine.blei@nyumc.org

$\equiv$ indicates article with supplemental on-line table.

DOI 10.3174/ajnr.A0937 pression, with $70 \%$ of children having only 1 extracutaneous manifestation. ${ }^{4}$ The most frequently seen extracutaneous manifestations are central nervous system (CNS) structural malformations and cerebrovascular anomalies, with incidences of $45 \%$ and $35 \%$, respectively. ${ }^{8}$ Typical malformations include Dandy-Walker complex and cerebellar hemisphere hypoplasia ipsilateral to the hemangioma. Less frequent findings include cortical dysplasia, dysplasia of the corpus callosum, and arachnoid cysts. ${ }^{10}$ Cerebrovascular anomalies in PHACES are predominantly the following: 1) hypoplasia or agenesis of major cerebral vessels, 2) persistence of embryonic vessels, 3) progressive vascular stenosis or occlusion, and/or 4) dolichoectasia of the cerebral vasculature. ${ }^{11}$

Recent studies report intracranial hemangiomas with a predilection for the cerebellopontine angle. ${ }^{12}$ Although not all of these abnormalities are clinically significant, the prevalence of neurologic sequelae among patients with PHACES and CNS structural or cerebrovascular anomalies ranges from $50 \%$ to $90 \%{ }^{8,13}$ Patients with PHACES are at risk for developmental delay, mental retardation, seizures, and infarcts, secondary to a progressive vasculopathy. ${ }^{14,15}$ Given the low incidence of PHACES, most reports are isolated case reports and small case series; however, a PHACES registry is underway (http://www.texaschildrenshospital.org/CareCenters/ Dermatology/Phace.aspx). Our current investigation is one of the largest combined institutional reviews of children with PHACES. We provide a detailed review of the neuroradiologic findings and the implications of these CNS anomalies.

\section{Methods}

This study was approved by the institutional review board. Additionally, parental consent for use of photographs was obtained. Clinical 


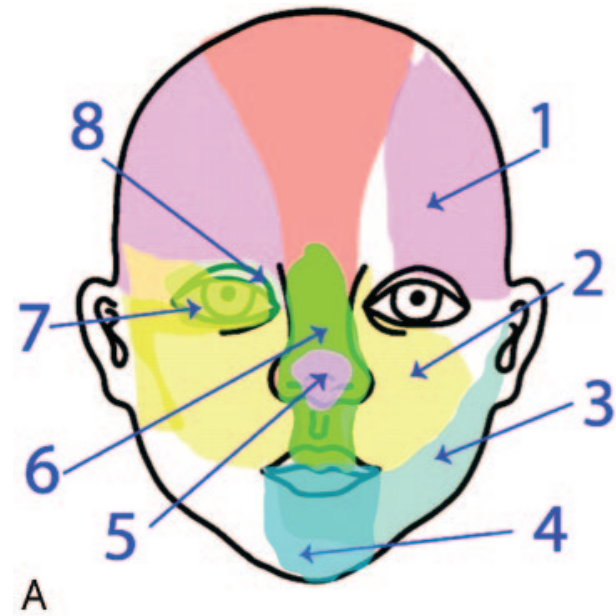

B
Fig 1. A, Eight initial embryonic developmental patterns are identified. $B$, Final segmental patterns are extracted from image analysis: S1 (frontotemporal), S2 (maxillary), S3 (mandibular), S4 (frontonasal). Reproduced with permission from J Pediatr 139:117-23, copyright 2001 by the AAP. records and imaging studies were reviewed in patients seen between 1998 and 2007 with a diagnosis of PHACES. All cases were initially referred for evaluation of facial hemangioma.

All patients underwent MR imaging; MR angiography (MRA); sternal x-ray; echocardiography; and ophthalmology, neurology, and cardiology consultations. Cutaneous mapping of the facial hemangiomas followed the segment map published by Haggstrom et al (Fig 1). "Beard" distribution hemangiomas included hemangiomas of the lower face and/or anterior neck previously described by Orlow et al. ${ }^{16}$ Neurodevelopmental and other sequelae were noted. MR imaging and MRA were repeated approximately every 6 months, and patients were seen at least monthly during their first year.

Imaging techniques included CT of the head, face, and neck; MR imaging of the brain, face, and neck; MRA; quantitative MRA (NonInvasive Optimal Vessel Analysis [NOVA]; VasSol, Chicago, Ill); and conventional angiography when clinically indicated. One hundred percent (17/17) of patients underwent MR imaging, and 76\% (13/17) underwent MRA. Eighty-two percent (14/17) of patients had repeat MR imaging, and 54\% (7/13) had repeat MRA. Two patients underwent head CT, and 2 patients underwent cerebral, head, and neck angiography. In 4 patients, NOVA scans provided quantitative flowrate assessment within the major cerebral vessels. Imaging studies were re-reviewed by a board-certified attending neuroradiologist.

\section{Results}

\section{Demographics}

Seventeen patients were identified with PHACES association (On-line Table). Fifteen of the 17 patients were female. The median age at first visit was 90 days. Follow-up was for 9 years for 1 patient and averaged 3.5 years for the entire sample. There were 9 white, 1 African-American, 1 Southeast Asian, and 6 Hispanic patients. Two patients were born prematurely, and 2 patients were products of in vitro fertilization.

\section{Hemangioma Distribution}

All but 1 patient (a patient with a deep facial hemangioma) had large segmental facial hemangiomas. Forty-seven percent $(n=8)$ of patients had left-sided hemangiomas, $18 \%(n=3)$ had right-sided hemangiomas, and $41 \%(n=7)$ of hemangiomas could be classified as beard distribution.

Complications from the hemangioma included the following: 1) airway obstruction (4 patients, 3 with beard distribu- tion); 2) ophthalmologic sequelae: amblyopia (3 patients), hyperopia (1 patient), and irreversible vision loss secondary to optic atrophy (1 patient); and 3 ) high-output congestive heart failure (1 patient).

Segmental mapping (based on patient photographs) showed the following: S1 distribution in 11 patients (65\%), S2 in $10(59 \%)$, S3 in $13(76 \%)$, and S4 in $6(35 \%)$ (On-line Table). Hemangiomas were restricted to only 1 segment in 6 patients ( 1 case involved S1 in isolation; 5 cases involved S3 in isolation). The most common hemangioma distribution was the S3-only pattern (Fig $2 A$ ), present in 5 patients (29\%); and the second most common was the S1-S4 hemangioma, present in 5 patients (29\%) (Fig 2B).

The association between extracutaneous anomalies and hemangioma distribution was assessed. Of the 8 patients with CNS structural abnormalities, $88 \%$ (7/8) had involvement of the S1 and S2 segments. Fifty percent of patients with structural malformations (4/8) and 50\% of patients with cerebrovascular anomalies (6/12) had large hemangiomas involving all 4 segments. Eighty percent (4/5) of patients with cardiac defects had hemangiomas involving the S3 segment. All patients with ocular anomalies had involvement of at least the S1 and S2 segments. Finally, 78\% (7/9) of patients with ventral developmental defects had S3 involvement. Among the 6 patients with beard distribution or S3-only hemangiomas, no patients had a CNS structural malformation, 2 patients had cerebrovascular anomalies, 2 patients had cardiac defects, no patients had ocular anomalies, and 5 patients had ventral developmental defects.

\section{CNS Structural Malformations}

Structural abnormalities were present in $50 \%$ of our patients, with 6 of the 8 patients having a posterior fossa malformation. The most common structural malformation was cerebellar hemispheric hypoplasia $(n=6)$. All cases of hypoplasia were ipsilateral to the hemangioma. Dandy-Walker variant was the second most common abnormality $(n=4)$, with all but 1 case associated with ipsilateral cerebellar hemisphere hypoplasia. Supratentorial malformations included cerebral cortical dysplasia $(n=2)$ and cerebral migrational abnormalities $(n=3)$. Cerebral cortical dysplasia involved the temporal and parietal lobes and a blurring of the gray matter-white matter junction. 

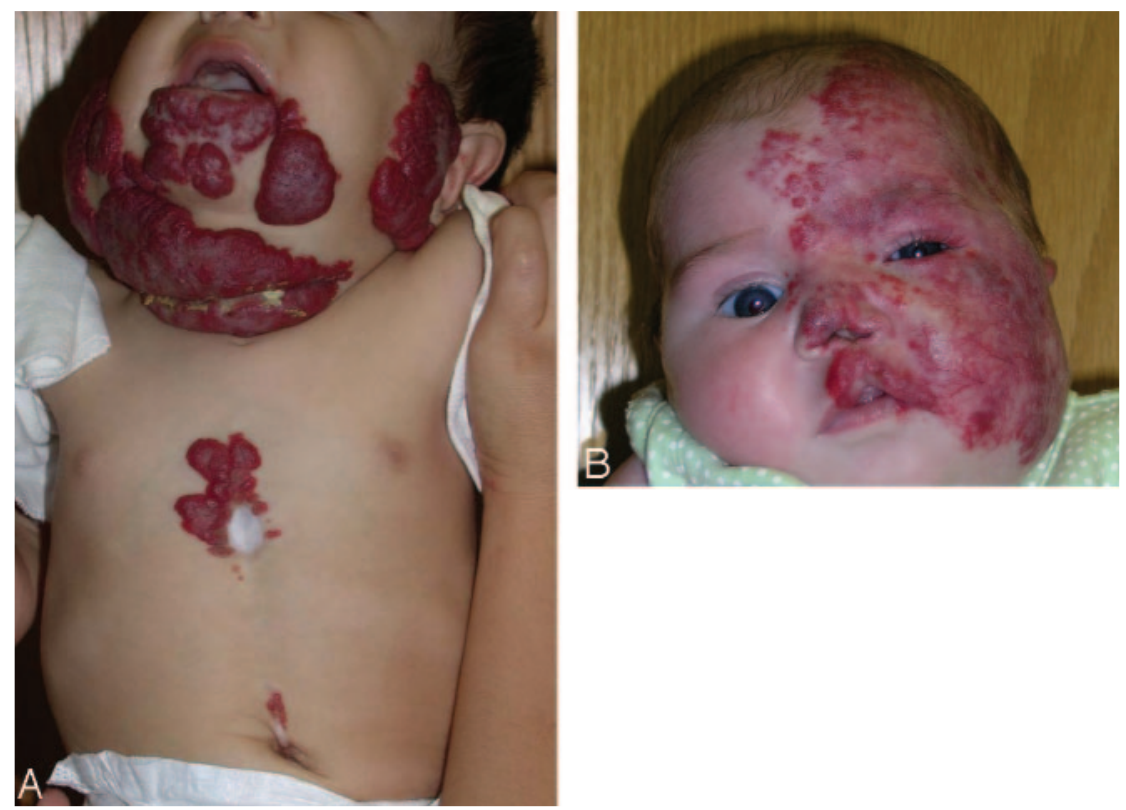

Fig 2. $A$, Color photograph of patient 10 , illustrating a hemangioma in an S3 distribution. Note additional thoracic and supraumbilical raphe. $B$, Color photograph of patient 1 with S1-S4 cutaneous distribution.
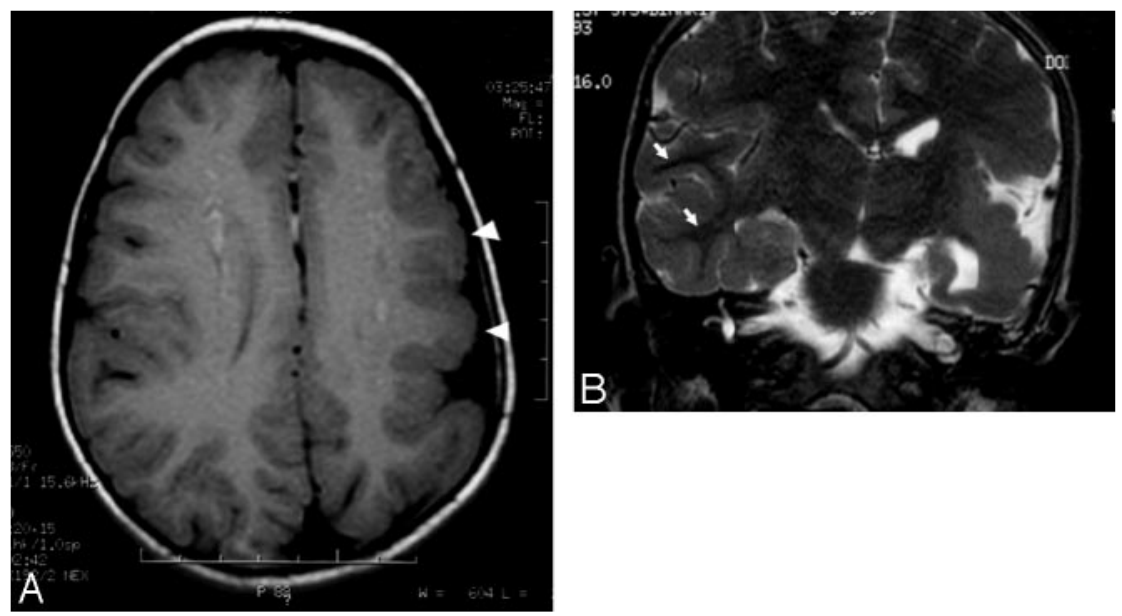

Fig 3. A, Axial T1-weighted image of the brain demonstrates decreased size of the left hemisphere and abnormally thickened left frontoparietal cortex (arrowheads). Also demonstrated is an abnormal pattern of sulcation with thickened gyri. $B$, Coronal T2-weighted image of the brain demonstrates prominent thickening of the left temporoparietal cortex with poor gray-white discrimination. Well-defined gray-white differentiation on the right (arrows) is marked for norma comparison.

Both patients with cerebral dysplasia also had posterior fossa abnormalities, and 1 of these 2 patients also had ipsilateral cerebral atrophy, which initially was misclassified as SturgeWeber syndrome. The migrational abnormalities included pachygyria, subependymal nodular gray matter heterotopia, and subcortical nodular gray matter heterotopia (Fig 3). Gray matter heterotopia was ipsilateral to the hemangioma in both cases. Additional structural malformations included cerebellar cortex dysplasia $(n=2)$, agenesis of the corpus callosum $(n=2)$, prominence of ipsilateral Meckel cave $(n=3)$, and arachnoid cyst $(n=1)$. All 8 patients with a structural malformation had at least 1 cerebrovascular anomaly.

\section{Cerebrovascular Anomalies}

MRA was used to assess the cerebral vasculature in 13 patients, with additional conventional angiography performed in 2 of these patients. Cerebrovascular anomalies were the most common type of extracutaneous manifestation detected (On-line Table). Tortuous dysplastic vessels were seen most commonly $(n=7)$ in the vertebrobasilar system $(6 / 7)$ and the internal carotid artery (ICA) (5/7) (Fig 4). The next most common anomalies were hypoplasia of the Al segment of the anterior cerebral artery $(n=5)$, the presence of anomalous vessels $(n=$ $4)$, and the persistence of embryonic vessels $(n=2)$. Persistent embryonic vessels included the trigeminal artery $(n=1)$ and the proatlantal artery and stapedial arteries in another patient. In 3 of the 4 cases of anomalous vessels, the vessels arose from the vertebrobasilar system.

Additional cerebrovascular anomalies included the absence of the ipsilateral ICA $(n=2)$, aneurysms $(n=2)$, narrowing of the ICA $(n=4)$, and narrowing of basilar artery $(n=1)$. Two patients exhibited interval changes of their cerebral vasculature on the basis of repeat imaging. One patient had regression of a left posterior cerebral artery P3 segment aneurysm demonstrated on serial conventional angiography. The other patient had progressive narrowing of the proximal ICAs bilaterally and developed a fusiform aneurysm on subsequent MRAs. Intracranial vascular lesions were detected by contrast MR imaging in 2 patients. One patient had leptomeningeal enhancement involving the anterior pons and the superior cerebellum ipsilateral to the hemangioma. The other patient had similar enhancement along the temporal lobes as well as having an enhancing nodular mass isointense to the 

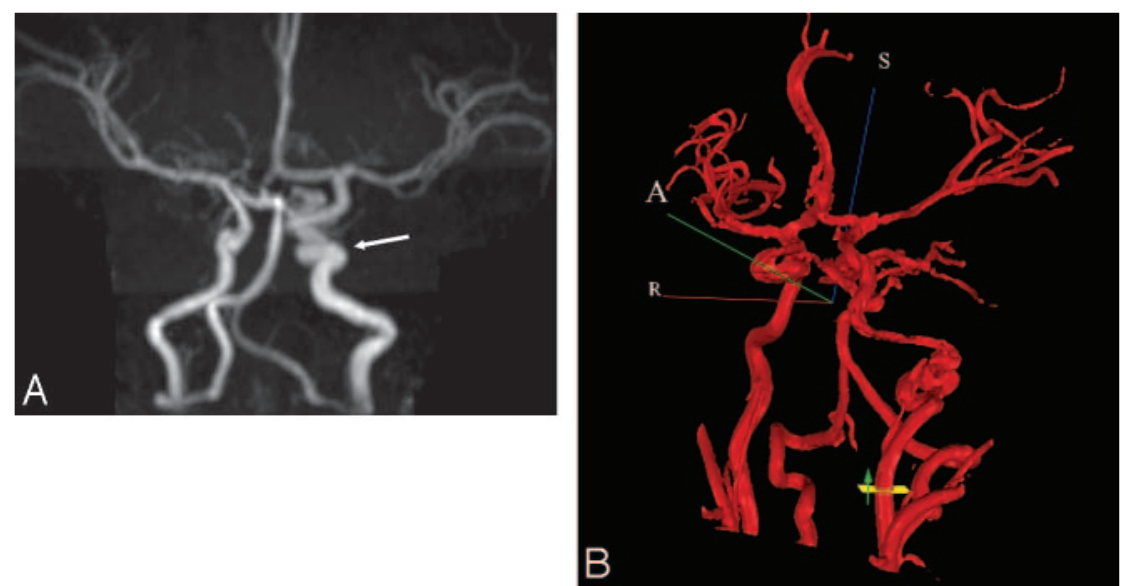

Fig 4. $A$, Coronal maximum-intensity-projection reconstruction from intracranial time-of-flight MRA demonstrates an ectatic and tortuous appearance of the left precavernous and cavernous ICA (arrow). B, 3D MRA rendering from NOVA with flow measurement through the cervical left ICA. The left ICA at the skull base is markedly irregular and tortuous.

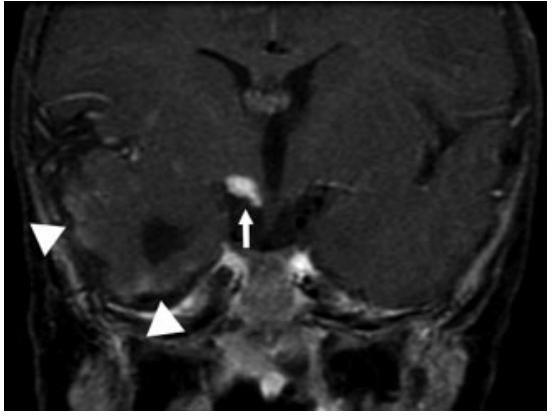

Fig 5. Postcontrast coronal T1-weighted image of the brain again demonstrates the abnormally enhancing mass (arrow) just below right-sided portions of the hypothalamus. Vague surface enhancement along the right temporal lobe is noted (arrowheads).

hemangioma anterior to a cerebral peduncle (Fig 5). Sixtyseven percent (8/12) of patients with cerebrovascular anomalies had underlying CNS structural malformations.

\section{Neurologic Sequelae}

Three patients developed significant neurologic symptoms constituting $25 \%$ of all patients with CNS structural malformations and/or cerebrovascular anomalies. Sequelae included hydrocephalus/Dandy-Walker malformation requiring shunt surgery (1 patient), global developmental delay (2 patients), and seizures (2 patients). All 3 patients with neurologic sequelae had congenital malformation of the cerebral cortex. The 2 patients with global developmental delay had a dysplastic cerebrum ipsilateral to the facial hemangioma. The 2 patients with seizures had heterotopic gray matter on MR imaging, with 1 of the patients also having cerebral dysplasia.

\section{Cardiovascular Malformations, Ocular Abnormalities, Ventral Developmental Defects, and Endocrine Abnormalities}

One third ( $n=5$ ) of our patients had cardiovascular anomalies, which included tetralogy of Fallot $(n=1)$, right-sided aortic arch $(n=1)$, ventricular septal defect requiring surgical repair $(n=1)$, common brachiocephalic trunk variant $(n=$ $1)$, aberrant left subclavian artery arising from the descending aorta $(n=1)$, and dilation of the aortic arch. ${ }^{1}$ One of the 5 patients with cardiac defects had a CNS structural and cerebrovascular malformation, and one had a cerebrovascular malformation with normal cranial structure. All 5 patients with cardiac defects had sternal/midline defects.
Although many patients had hemangioma-related ptosis or visual axis obstruction, $24 \%$ had a congenital ocular abnormality. The abnormalities included contralateral cataract $(n=$ $1)$, ipsilateral iris hemangioma $(n=1)$, ipsilateral Duane syndrome $(n=1)$, and contralateral morning glory disk anomaly $(n=1)$. Three of the 4 patients with ocular abnormalities had both CNS structural and cerebrovascular anomalies, and the other patient with ocular abnormalities had cerebrovascular abnormalities with a normal brain structure. The patient with cataract in the contralateral eye developed secondary glaucoma ipsilateral to multiple hypervascular hemangiomas and venous hypertension. In this patient, the restricted cerebral and craniofacial venous system competed with the increased flow of the multiple hemangiomas.

Fifty-three percent of patients $(n=9)$ had a ventral developmental defect, such as suprasternal skin abnormalities ( $n=$ $5)$, hypersegmented sternal ossification centers $(n=4)$, hypopigmented vertical streaks or supraumbilical raphe $(n=3)$, upper lip ipsilateral and nares clefting ipsilateral to the hemangioma $(n=2)$ and cleft soft palate $(n=1)$, and congenital hypothyroidism $(n=1)$.

\section{Discussion}

PHACES is an underappreciated and underdiagnosed neurocutaneous association, which may be as common as SturgeWeber syndrome. ${ }^{8,17}$ The constellation of PHACES criteria per patient may be highly variable. Our investigation of 17 patients focused specifically on the cerebrovascular and CNS structural malformations and broadened the spectrum of this disease

\section{CNS Structural Malformations}

The association between CNS structural malformations and segmental hemangiomas has been reported in the literature since $1978 .{ }^{3}$ Between $43 \%$ and $90 \%$ of patients with PHACES have a CNS structural malformation..$^{4,8,13,18,19}$ Malformations typically involve the posterior fossa, presenting as a DandyWalker complex, isolated cerebellar hemispheric hypoplasia, or a combination of the 2; our sample was similar in this regard. Half of our patients had a CNS structural malformation. Three quarters of these patients had a posterior fossa abnormality, with the most common abnormality being a DandyWalker variant with cerebellar hemispheric hypoplasia ipsilateral to the facial hemangioma. Many previous reports on 
PHACES have failed to distinguish between Dandy-Walker variants (hypogenesis of the cerebellar vermis and cystic dilation of the fourth ventricle without enlargement of the posterior fossa) and Dandy-Walker malformations (enlarged posterior fossa, hypogenesis of the cerebellar vermis, and cystic dilation of the fourth ventricle). Only 1 child in our sample had a true Dandy-Walker malformation. These findings suggest that many cases of posterior fossa anomalies within PHACES are likely Dandy-Walker variants, and the patients are, therefore, at a lower risk of developing obstructive hydrocephalus.

Although posterior fossa anomalies define the PHACES spectrum, supratentorial cortical malformations are an under-recognized manifestation of this disorder. To our knowledge, only 4 cases have been published describing supratentorial cortical malformations. ${ }^{10,20,21}$ The authors of these 3 articles reported cases of cortical dysplasia limited to the frontal region, subependymal heterotopic gray matter, and polymicrogyria and a case of mild holoprosencephaly. In each of the previous cases, the cortical malformations occurred in the absence of posterior fossa anomalies or other manifestations of PHACES, leading to the conjecture that these patients may represent a separate neurocutaneous disorder. ${ }^{10}$

Within our sample, 4 patients exhibited supratentorial cortical malformations. The malformations detected included isolated polymicrogyria, cortical dysplasia with polymicrogyria and posterior parietal cleft, cortical hypoplasia and dysplasia of the temporal and parietal lobe with pachygyria and subcortical nodular heterotopia, and an isolated case of subependymal nodular heterotopia. All 4 patients had posterior fossa anomalies consistent with PHACES, and cortical dysplasia and heterotopic gray matter were always ipsilateral to the facial hemangiomas. The types of cortical dysgenesis detected in our sample included abnormalities of cortical organization and migrational abnormalities. Furthermore, the detection of a cortical cleft is a subtle radiologic finding associated with cortical dysgenesis in $40 \%$ of patients. ${ }^{22}$ In most cases of cortical dysgenesis, the exact etiology often remains unknown.

Additionally, the coexistence of CNS structural malformations and cerebrovascular anomalies was very common. One hundred percent of patients with a structural malformation had a cerebrovascular abnormality, and $75 \%$ of patients with a cerebrovascular anomaly had an extracerebral structural malformation. Other authors have also found high rates of coexistence. Pascual-Castroviejo et $\mathrm{al}^{18}$ documented that $88 \%$ of patients with structural malformations had cerebrovascular anomalies and $64 \%$ of patients with a cerebrovascular anomaly had a CNS structural malformation. In another large review of patients with PHACES, coexistence was found in only $30 \%$ of patients. ${ }^{8}$ High rates of coexistence raise the possibility of a common process leading to cerebral vascular anomalies, posterior fossa malformations, and cortical dysgenesis in PHACES. Recent work in primitive animal models, such as the zebrafish, has shown that both vascular and neuronal pathfinding and patterning may share similar signaling pathways, in particular the semaphorin-plexin signaling pathway. ${ }^{23} \mathrm{Ad}-$ ditional basic science research is needed to support the clinical findings that cerebrovascular and neuronal organization codevelops and may share conserved mechanisms for patterned development.
Beyond serving as an additional indicator of PHACES, cortical malformations hold neurologic significance, given their epileptogenic potential-10\%-40\% of patients with intractable seizures have evidence of cortical dysplasia on MR imaging. ${ }^{24-27}$ All 3 patients in our sample who developed significant neurologic sequelae had evidence of cortical dysgenesis. The 2 patients with global developmental delay (language $>$ motor) had cortical dysplasia, and the 2 patients who developed seizures had focal nodular heterotopia on MR imaging. Clinicians should recognize that any child with PHACES who develops seizures should have MR imaging to rule out the possibility of stroke and abnormalities of cortical migration. Furthermore, the detection of areas of cortical dysgenesis is important in the setting of intractable seizures because surgical resection can be a curative therapy in certain cases. To date, posterior fossa anomalies have been emphasized as an indicator of PHACES; however, others emphasize that PHACES is likely a disorder that affects CNS development above and below the tentorium and that each child with PHACES should be evaluated for errors in cortical development. ${ }^{10}$

\section{Cerebrovascular Anomalies}

Case series have reported that approximately half of all patients with PHACES have a cerebrovascular anomaly. ${ }^{4,8,13,18,19}$ Rates may be even higher because vascular imaging may not be performed on every patient with PHACES. Within our sample, 13 of 17 patients underwent an MRA. Twelve of these 13 patients were found to have some degree of anomalous vasculature, underscoring the importance of MRAs and other vascular studies within this population. Five different types of vascular lesions have been reported in patients with PHACES and were likewise found within our sample: persistence of embryonic vessels (typically, trigeminal, hypoglossal, and proatlantal arteries), agenesis of major cervical arteries, anomalous vasculature, progressive arterial stenosis, and segmental dolichoectasia. ${ }^{11}$ The vascular abnormalities are consistently ipsilateral to the facial hemangioma and often involve the carotid or vertebrobasilar system in a segmental fashion. The most common abnormality detected was tortuous cerebral vasculature. Although tortuous cervical and intracranial arteries may be seen in young children, in PHACES the vessels are also dysplastic with aneurysmal dilations and frequently are embryonal and/or have an abnormal course. Recent publications have highlighted the findings that some patients with PHACES are at risk for developing a progressive vasculopathy in which vessels become further dilated and tortuous, develop Moyamoya-type collaterals, and may eventually undergo complete occlusion. These patients are at increased risk of stroke, and strokes have been described in children as young as 4 months of age and as old as 14 years of age. ${ }^{15,28}$ None of our patients developed ischemic strokes. However, 2 patients did have documented progressive vascular changes, and tortuous vasculature was very common within our sample. Both carotid and vertebrobasilar vessels were noted to have a tortuous and, at times, corkscrew-like appearance in our sample. Additional studies are needed to further assess whether vessel tortuosity correlates with subsequent vaso-occlusion. 


\section{CNS Intracranial and Surface Enhancement}

Contrast-enhancing CNS masses in patients with PHACES, consistent with intracranial hemangiomas, have been reported in several recent articles..$^{3,4,12,15,19,29,30}$ The intracranial hemangiomas were located in the internal auditory canal and cerebellopontine angle as reported by Judd et $\mathrm{al}^{31}$ and these lesions involuted in parallel with the facial hemangiomas. One of our patients had a contrast-enhancing mass anterior to the cerebral peduncle that was isointense with the facial hemangioma. This had been radiologically stable and clinically asymptomatic. This patient also had leptomeningeal enhancement along the right temporal lobe. One other patient had this enhancement along the superior cerebellum and anterior pons. This has been reported in only 2 other cases. ${ }^{12,19}$ Intracranial hemangiomas are uncommon in children. As with other hemangiomas of infancy, it is anticipated that these will involute, and this change can be documented with repeat MR imaging.

\section{Hemangioma Segmental Analysis}

The first clues toward understanding the underlying pathology of PHACES may be found in the anatomic distribution of the facial hemangiomas. These are often large and plaquelike in a geographic pattern. The literature suggests that infantile hemangiomas do not correspond with facial dermatomes or lines of Blaschko, patterns of neuroectodermal migration. Focal-type hemangiomas, hemangiomas with a tumorlike appearance, map to areas of the face in close proximity to lines of mesenchymal or mesenchymal-ectodermal embryonic fusion. $^{2}$ Diffuse hemangiomas, the type characteristic of PHACES, have a segmental distribution corresponding, partially, to developmental facial prominences. ${ }^{1}$

The hemangiomas of patients in our sample were mapped to the nomenclature provided by Haggstrom et $\mathrm{al}^{1}$ and Waner el al. ${ }^{2}$ We found most hemangiomas mapped to 1 of 3 distributions: hemifacial, frontotemporal, or the mandibular region. Segment S4 (frontonasal segment) and segment S2 (maxillary segment) never occurred in isolation. Metry et $\mathrm{al}^{8}$ also found a lack of isolated S2 and S4 hemangiomas and found both segments frequently coexisting with S1 segment involvement. Additional studies are needed to assess whether our findings merely reflect a lack of sufficient sample size or instead point toward a map of segmental hemangiomas that consist of only 2 segments: S3 (mandibular segment) and a combined S1, S4, and S2 segment (frontotemporal, nasal, and maxillary segments) with variable expression.

Hemangioma location holds clinical relevance in PHACES because according to Metry et al, ${ }^{8}$ hemangiomas involving the upper half of the face (S1 and S4) correlated with structural brain, cerebrovascular, and ocular anomalies. Furthermore, hemangiomas in a mandibular (S3) distribution were associated with ventral developmental defects, such as sternal abnormalities and supraumbilical raphe. Our current sample corroborates these findings. The S1 segment was involved in $83 \%$ of patients with CNS or cerebrovascular anomalies and $100 \%$ of patients with ocular abnormalities. Furthermore, it appears that the size of the hemangioma also was associated with the likelihood of CNS structural or cerebrovascular abnormalities. Large hemangiomas that involved S1 through S3 and/or S4 comprised 75\% of the patients with CNS structural malformations and $58 \%$ of the patients with cerebrovascular anomalies.

Hemangiomas only involving the mandibular, S3, segment or beard-distribution hemangiomas were closely associated with subglottic or upper airway hemangiomas in $40 \%$ of patients, cardiovascular anomalies in $20 \%$, and ventral developmental findings in $80 \%$. These hemangiomas were infrequently found with cerebral vascular anomalies (1 of 5 patients) and never with CNS structural malformations. Neural crest cells of a more rostral origin in the developing embryo compose the mesenchyme in the frontotemporal region of the face, whereas crest cells of a more caudal origin contribute the developing mandibular segment. This topographic distribution also reflects the neural crest contributions to different structures affected by PHACES, because the neural crest cells involved in cardiogenesis have a more caudal origin than the crest cells that contribute to the cerebrovascular walls. Larger studies are needed to help further define these associations and elucidate the potential role of neural crest cells in PHACES.

As with many of the previously published studies, our small sample size makes it difficult to draw wide-ranging conclusions. Larger studies are necessary to provide statistical power to correlate the coexistence of facial hemangioma distribution and extracutaneous manifestations. Also, it is unclear whether certain radiologic findings in patients with PHACES reflect true expressions of the disorder or are, in fact, normal variants. This is especially true with regard to vascular imaging. There are no published normative data for infant MRAs; therefore; it is unknown whether some findings in PHACES, such as vessel tortuosity, are normally seen in this age group. However, in our experience with cerebral conventional angiography for other pediatric disorders, we believe that abnormalities such as those described in this PHACES population are infrequent. Studies have shown that up to $13 \%$ of the population may have A1 segment hypoplasia, and this is considered a normal variant. $^{32}$ We observed a higher incidence in our patients.

Our data support and expand the work of others in identifying risk factors for associations with segmental hemangiomas. Patients with large facial cutaneous segmental (S1-S4, especially S1) hemangiomas are most at risk of CNS structural and cerebrovascular anomalies, S1 with ocular anomalies and S3 with airway, ventral, and cardiac anomalies. Patients with both complex CNS structural anomalies as well as complex CNS vasculopathies seem to be at most risk for developmental and/or other neurologic sequelae (eg, seizures). We continue to follow all patients with CNS arteriopathies clinically and with serial MRA examinations. The long-term prognosis of these patients remains unknown. Longitudinal studies (including measures of cognitive and motor development and school performance) are imperative to monitor the natural history of patients with PHACES. This will facilitate 1) identification of those at highest risk for stroke and/or seizures, 2) establishment of guidelines for monitoring, 3) suitable ancillary support for these patients, and 4) evidence-based realistic expectations and counseling for families. Additionally, future studies to assess the efficacy of antithrombotic therapies in stroke prevention for those at highest risk should also be considered. 


\section{References}

1. Haggstrom AN, Lammer EJ, Schneider RA, et al. Patterns of infantile hemangiomas: new clues to hemangioma pathogenesis and embryonic facial development. Pediatrics 2006;117:698-703

2. Waner M, North PE, Scherer KA, et al. The nonrandom distribution of facial hemangiomas. Arch Dermatol 2003;139:869-75

3. Pascual-Castroviejo I. Vascular and nonvascular intracranial malformation associated with external capillary hemangiomas. Neuroradiology 1978;16:82-84

4. Frieden IJ, Reese V, Cohen D. PHACE syndrome: the association of posterior fossa brain malformations, hemangiomas, arterial anomalies, coarctation of the aorta and cardiac defects, and eye abnormalities. Arch Dermatol 1996;132:307-11

5. Pascual-Castroviejo I, Pascual-Pascual SI, Rafia S, et al. Hemangiomas, and cutaneous and intracranial vascular deformations (Pascual-Castroviejo syndrome type II): a case report [in Spanish]. Rev Neurol 2002;35:1034-36

6. Pascual-Castroviejo I. "New" 3C syndrome. Dev Med Child Neurol 1993;35:1026-27

7. Goh WH, Lo R. A new 3C syndrome: cerebellar hypoplasia, cavernous haemangioma and coarctation of the aorta. Dev Med Child Neurol 1993;35:637-41

8. Metry DW, Haggstrom AN, Drolet BA, et al. A prospective study of PHACE syndrome in infantile hemangiomas: demographic features, clinical findings, and complications. Am J Med Genet A 2006;140:975-86

9. Hersh JH, Waterfill D, Rutledge J, et al. Sternal malformation/vascular dysplasia association. Am J Med Genet 1985;21:177-86, 201-02

10. Grosso S, De Cosmo L, Bonifazi E, et al. Facial hemangioma and malformation of the cortical development: a broadening of the PHACE spectrum or a new entity? Am J Med Genet A 2004;124:192-95

11. Baccin CE, Krings T, Alvarez H, et al. A report of two cases with dolichosegmental intracranial arteries as a new feature of PHACES syndrome. Childs Nerv Syst 2007;23:559-67. Epub 2006 Oct 13

12. Tortori-Donati P, Fondelli MP, Rossi A, et al. Intracranial contrast-enhancing masses in infants with capillary haemangioma of the head and neck: intracranial capillary haemangioma? Neuroradiology 1999;41:369-75

13. Metry DW, Dowd CF, Barkovich AJ, et al. The many faces of PHACE syndrome. J Pediatr 2001;139:117-23

14. Heyer GL, Millar WS, Ghatan S, et al. The neurologic aspects of PHACE: case report and review of the literature. Pediatr Neurol 2006;35:419-24

15. Bhattacharya JJ, Luo CB, Alvarez $\mathrm{H}$, et al. PHACES syndrome: a review of eight previously unreported cases with late arterial occlusions. Neuroradiology 2004;46:227-33

16. Orlow SJ, Isakoff MS, Blei F. Increased risk of symptomatic hemangiomas of the airway in association with cutaneous hemangiomas in a "beard" distribution. J Pediatr 1997;131:643-46

17. Pascual-Castroviejo I, Pascual-Pascual SI, Delgado J. Cutaneous hemangiomas and vascular abnormalities: persistence of embryonic vascularization. AJNR Am J Neuroradiol 2007;28:390-91

18. Pascual-Castroviejo I, Viano J, Moreno F, et al. Hemangiomas of the head, neck, and chest with associated vascular and brain anomalies: a complex neurocutaneous syndrome. AJNR Am J Neuroradiol 1996;17:461-71

19. Poetke M, Frommeld T, Berlien HP. PHACE syndrome: new views on diagnostic criteria. Eur J Pediatr Surg 2002;12:366-74

20. Pascual-Castroviejo I, Viano J, Pascual-Pascual SI, et al. Facial haemangioma, agenesis of the internal carotid artery and dysplasia of cerebral cortex: case report. Neuroradiology 1995;37:692-95

21. Aeby A, Guerrini R, David P, et al. Facial hemangioma and cerebral corticovascular dysplasia: a syndrome associated with epilepsy. Neurology 2003;60:1030-32

22. Bronen RA, Spencer DD, Fulbright RK. Cerebrospinal fluid cleft with cortical dimple: MR imaging marker for focal cortical dysgenesis. Radiology 2000;214:657-63

23. Torres-Vazquez J, Gitler AD, Fraser SD, et al. Semaphorin-plexin signaling guides patterning of the developing vasculature. Dev Cell 2004;7:117-23

24. Guerrini R, Dravet C, Raybaud C, et al. Epilepsy and focal gyral anomalies detected by MRI: electroclinico-morphological correlations and follow-up. Dev Med Child Neurol 1992;34:706-18

25. Kuzniecky RI. Neuroimaging in pediatric epilepsy. Epilepsia 1996;37(suppl 1):S10-21

26. Palmini A, Gambardella A, Andermann F, et al. Operative strategies for patients with cortical dysplastic lesions and intractable epilepsy. Epilepsia 1994; 35(suppl 6):S57-71

27. Jackson GD. New techniques in magnetic resonance and epilepsy. Epilepsia 1994;35(suppl 6):S2-13

28. Burrows PE, Robertson RL, Mulliken JB, et al. Cerebral vasculopathy and neurologic sequelae in infants with cervicofacial hemangioma: report of eight patients. Radiology 1998;207:601-07

29. Ersoy S, Mancini AJ. Hemifacial infantile hemangioma with intracranial extension: a rare entity. Pediatr Dermatol 2005;22:309-13

30. Poindexter G, Metry DW, Barkovich AJ, et al. PHACE syndrome with intracerebral hemangiomas, heterotopia, and endocrine dysfunction. Pediatr Neurol 2007;36:402-06

31. Judd CD, Chapman $P R$, Koch $B$, et al. Intracranial infantile hemangiomas associated with PHACE syndrome. AJNR Am J Neuroradiol 2007;28:25-29

32. Chuang YM, Liu CY, Pan PJ, et al. Anterior cerebral artery Al segment hypoplasia may contribute to A1 hypoplasia syndrome. Eur Neurol 2007;57: $208-11$ 